\title{
Fusiform Aneurysm Associated With Fenestration of the Posterior Communicating Artery -Case Report-
}

\author{
Shiro BABA, Yuutaka FUKUDA, Shingo MIZOTA, \\ Kentaro HAYASHI, Kazuhiko SUYAMA, and Izumi NAGATA \\ Department of Neurosurgery, Nagasaki University Graduate School of \\ Biomedical Sciences, Nagasaki
}

\begin{abstract}
A 62-year-old male presented with a rare case of fenestration of the supraclinoid segment of the internal carotid artery (ICA) at the origin of the posterior communicating artery (PCoA). The patient had a fusiform aneurysm at the proximal branch of the PCoA, which was successfully clipped, sparing the anterograde blood flow. The double origin and fenestration of the PCoA branching off at the $\mathrm{C}_{2}$ segment of the left ICA suggested that this anomalous fenestration might have developed as the origin of the PCoA rather than the supraclinoid ICA during the early embryonal stage.
\end{abstract}

Key words: fenestration, posterior communicating artery, internal carotid artery, intracranial aneurysm, three-dimensional digital subtraction angiography

\section{Introduction}

Fenestrations of the intracranial arteries are sometimes associated with cerebral aneurysms, most commonly in the anterior communicating artery and the vertebrobasilar arteries, but also sporadically in the supraclinoid segment of the ICA at origin of the posterior communicating artery (PCoA). ${ }^{1-3,5-8,10-13)}$ We describe an extremely rare case of a fenestration that possibly consists of a double origin of the PCoA with an associated fusiform aneurysm.

\section{Case Report}

A 62-year-old male was referred to our hospital for a cerebral aneurysm incidentally found during a brain check-up at a local clinic. Magnetic resonance angiography showed an aneurysm at the $\mathrm{C}_{2}$ segment of the left ICA. He had a history of hypertension and hyperlipidemia. On admission, he had no symptoms or neurological signs. Three-dimensional digital subtraction angiography (DSA) revealed a fusiform aneurysm, $6 \mathrm{~mm}$ in maximum diameter, arising from the $\mathrm{C}_{2}$ segment of the left ICA with the dome projecting inferomedially, and the left fetal-type PCoA branching and coursing inferolaterally. In addition, a smaller artery branched off just distal to the aneurysmal neck, and joined the larger artery to form the main trunk of the PCoA (Fig. 1).

Left frontotemporal craniotomy was performed. Partial resection of the anterior clinoid process and opening of the distal dural ring exposed part of the aneurysm project-

Received October 22, 2009; Accepted January 5, 2010
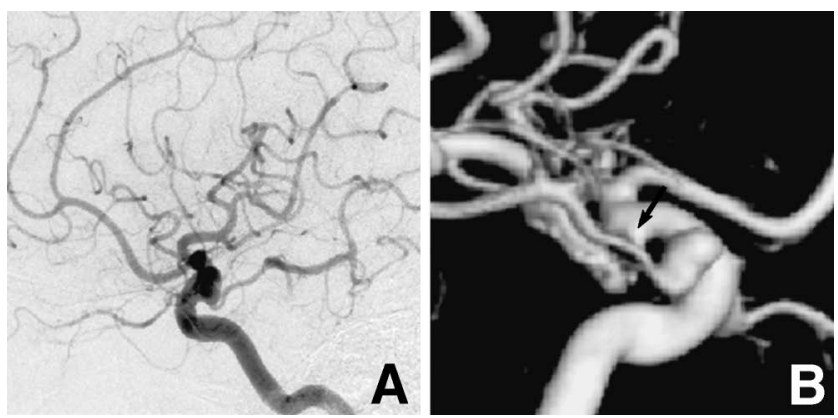

Fig. 1 (A) Left internal carotid angiogram, lateral view, showing a saccular aneurysm at the $\mathrm{C}_{2}$ segment of the left internal carotid artery (ICA). (B) Three-dimensional digital subtraction angiogram showing the larger branch originating from the dome, and the smaller branch (arrow) originating from the ICA just distal to the aneurysm neck, which joined the larger branch to form the main trunk of the posterior communicating artery.

ing inferomedially at the $\mathrm{C}_{2}$ segment of the ICA. By mobilizing the ICA medially, the larger branch originating from the dome and transforming smoothly to the main trunk of the PCoA was confirmed. This larger branch was approximately double the diameter of the smaller branch, which joined laterally to the larger one. The perforating arteries of the PCoA arose just distal to this junction (Fig. $2 \mathrm{~A}, \mathrm{~B})$. The main part of the aneurysm dome was located medially to the ICA, and no perforating arteries arose from the proximal site of the PCoA near the dome (Fig. 2C, D). Clipping of the aneurysm dome was difficult with preservation of the larger proximal branch of the PCoA, so 

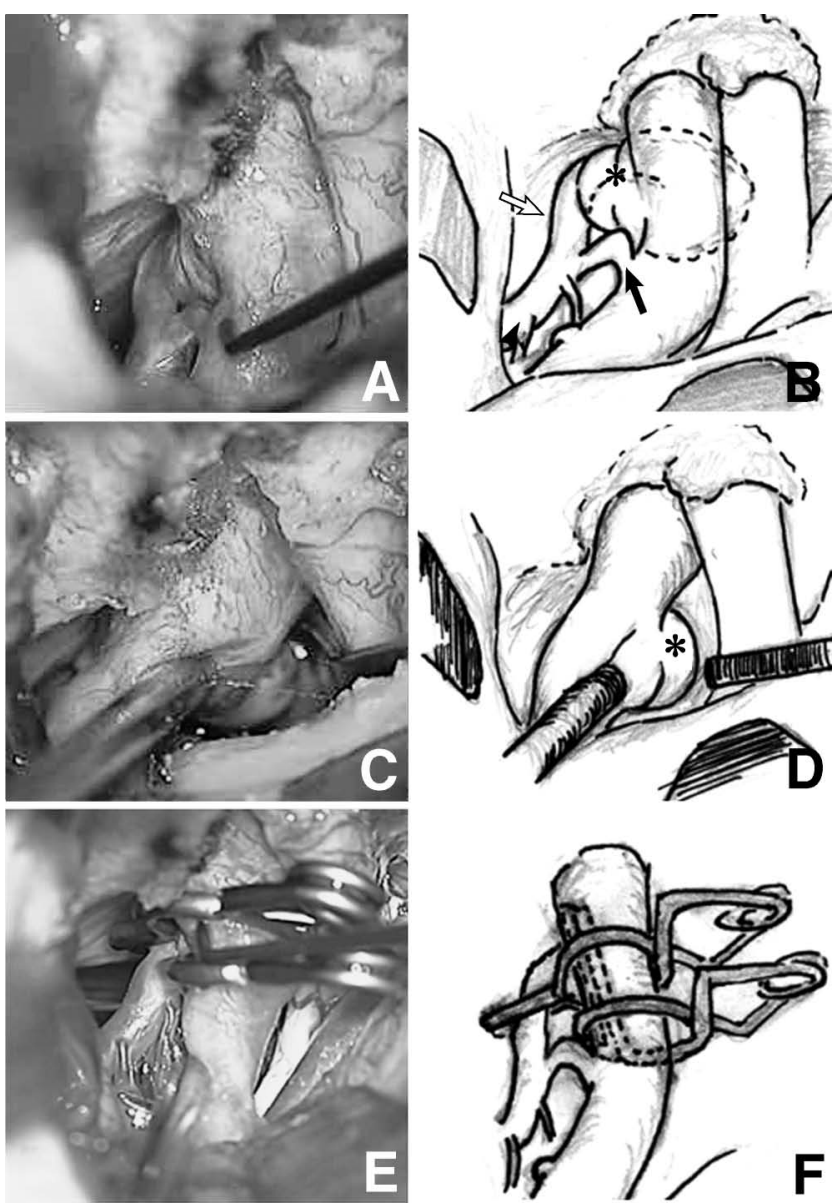

Fig. 2 Intraoperative photograph (A) and illustration (B) showing a fusiform aneurysm (asterisk), the larger branch (open arrow), the smaller branch (arrow), and the main trunk of the posterior communicating artery (PCoA) (arrowhead). The larger branch originates from the dome, smoothly transforming to the main trunk of the PCoA. The smaller branch originating from the internal carotid artery (ICA) just distal to the aneurysm neck joins laterally to the larger branch. Intraoperative photograph (C) and illustration (D) showing the main part of the aneurysm dome (asterisk) medial to the ICA and no perforating arteries. Intraoperative photograph (E) and illustration (F) showing the two fenestrated clips applied to obliterate the aneurysm neck and larger proximal branch, thus preserving anterograde blood flows in the main trunk of the PCoA and its perforating arteries.

a right-angled fenestrated clip was applied to the neck of the aneurysm. The larger branch of the PCoA was then obliterated with a straight fenestrated clip to prevent retrograde flow into the aneurysm from the PCoA (Fig. 2E, F). Anterograde blood flow in the main trunk of the PCoA and the perforating arteries was confirmed with micro-Doppler sonography.

Postoperative DSA showed obliteration of the aneurysm and preservation of the main trunk of the PCoA (Fig. 3). The patient was discharged 8 days after surgery without sequelae.

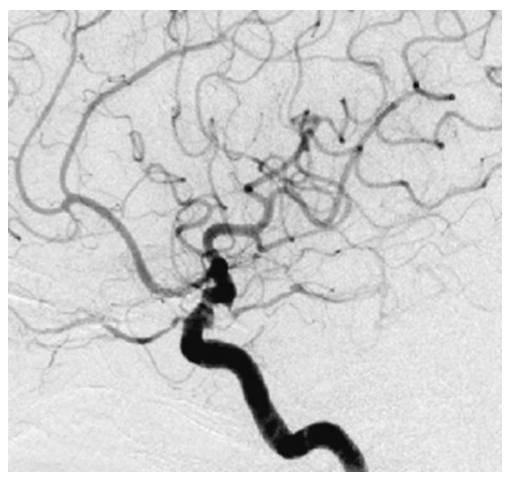

Fig. 3 Postoperative left internal carotid angiogram showing obliteration of the aneurysm and preservation of the main trunk of the posterior communicating artery.

Table 1 Summary of cases of fenestration of the supraclinoid internal carotid artery (ICA) or the posterior communicating artery (PCoA)

\begin{tabular}{|c|c|c|c|c|}
\hline Author (Year) & $\begin{array}{c}\text { Age } \\
\text { (yrs)/ } \\
\text { Sex }\end{array}$ & Side & $\begin{array}{c}\text { Associated } \\
\text { aneurysm }\end{array}$ & $\mathrm{SAH}$ \\
\hline \multicolumn{5}{|c|}{ Fenestration of the supraclinoid ICA: } \\
\hline Yock $(1984)^{13)}$ & $41 / \mathrm{F}$ & $\mathrm{rt}$ & + & + \\
\hline Findlay et al. $(1987)^{3)}$ & $28 / \mathrm{F}$ & $\mathrm{rt}$ & + & + \\
\hline Takano et al. $(1991)^{10)}$ & $51 / \mathrm{F}$ & $\mathrm{rt}$ & - & - \\
\hline $\begin{array}{l}\text { Hattori and Kobayashi } \\
(1992)^{5)}\end{array}$ & $38 / \mathrm{F}$ & lt & + & + \\
\hline Banach and Flamm (1993) $)^{1)}$ & $37 / \mathrm{F}$ & lt & + & + \\
\hline Katsuta et al. $(1993)^{6)}$ & $47 / \mathrm{F}$ & rt & - & + \\
\hline $\mathrm{Ng}$ et al. $(2006)^{7)}$ & $34 / \mathrm{F}$ & $\mathrm{rt}$ & + & + \\
\hline Chen et al. $(2007)^{2)}$ & $31 / \mathrm{M}$ & bil & + & + \\
\hline Onoda et al. $(2008)^{8)}$ & $42 / \mathrm{F}$ & lt & + & - \\
\hline \multicolumn{5}{|l|}{ Fenestration of the PCoA: } \\
\hline Yasargil (1984) $)^{12)}$ & NA & $\mathrm{rt}$ & + & - \\
\hline Tripathi et al. $(2003)^{11)}$ & $21 / \mathrm{M}$ & $\mathrm{rt}$ & - & - \\
\hline Present case & $62 / \mathrm{M}$ & lt & + & - \\
\hline
\end{tabular}

NA: not available, SAH: subarachnoid hemorrhage.

\section{Discussion}

Only a few cases of fenestrations of the intracranial arteries in the supraclinoid segment of the ICA and the PCoA have been reported (Table 1). ${ }^{1-3,5-8,10-13)}$ The common finding is that the fenestration is located just distal to the branching site of the ophthalmic artery. However, the definitions that distinguish the site of fenestration between the ICA and the PCoA have not yet been clearly described.

Fenestration of the cerebral arteries presumably results from anomalous fusion or division of primitive vessels. The primitive carotid artery arises from the third aortic arch at the $4 \mathrm{~mm}$ to $5 \mathrm{~mm}$ embryological stage, and then divides to form the cranial and caudal divisions of the ICA just distal to the ophthalmic artery. ${ }^{9)}$ The cranial division gives rise to the anterior choroidal, middle cerebral, and anterior cerebral arteries, whereas the caudal division 
terminates as the PCoA. The intracranial ICAs temporarily connect to each other through small plexiform channels. $^{3,9)}$ An anomalous branching or fenestration may occur at the division of the primitive ICA, where the ophthalmic artery originates just proximally. ${ }^{1,3,4,8-10,13)}$ In our case, two distinct vessels with different diameters branched off and smoothly fused together into the main trunk of the PCoA. These findings suggested that this anomalous fenestration might have developed as the origin of the PCoA rather than the supraclinoid ICA during the early embryonal stage.

In the present case, two clips were applied to obliterate the aneurysm, so preserving the anterograde blood flows in the main trunk of the PCoA and its perforating arteries. In general, thorough inspection of the structure is essential before applying clips to such a complicated aneurysm. Since the anterior thalamoperforating artery arises from the main trunk of the PCoA, the blood flow of the PCoA must be preserved. Preoperative three-dimensional DSA can be useful to demonstrate the anatomical variations precisely, and thus determine the surgical strategy. In addition, the patency of the PCoA and its perforating arteries should be confirmed intraoperatively using micro-Doppler sonography or an alternative procedure such as intraoperative DSA and indocyanine green-video angiography.

\section{References}

1) Banach MJ, Flamm ES: Supraclinoid internal carotid artery fenestration with an associated aneurysm. J Neurosurg 79: 438-441, 1993

2) Chen YY, Chang FC, Hu HH, Chao AC: Fenestration of the supraclinoid internal carotid artery associated with aneurysm and ischemic stroke. Surg Neurol 68: 60-63, 2007
3) Findlay JM, Chui M, Muller PJ: Fenestration of the supraclinoid internal carotid artery. Can J Neurol Sci 14: 159-161, 1987

4) Hashimoto M, Urasaki E, Tsujigami S, Yokota A: Ruptured aneurysm associated with partially duplicated posterior communicating artery. Neurol Med Chir (Tokyo) 42: 23-26, 2002

5) Hattori T, Kobayashi H: Fenestration of the supraclinoid internal carotid artery associated with carotid bifurcation aneurysm. Surg Neurol 37: 284-288, 1992

6) Katsuta T, Matsubara T, Fujii K: Fenestration of the supraclinoid internal carotid artery. Neuroradiology 35: 461, 1993

7) Ng PP, Steinfort B, Stoodley MA: Internal carotid artery fenestration with dual aneurysm. J Neurosurg 104: 979, 2006

8) Onoda K, Ono S, Tokunaga K, Sugiu K, Date I: Fenestration of the supraclinoid internal carotid artery with associated aneurysm. Neurol Med Chir (Tokyo) 48: 118-120, 2008

9) Paget DH: The development of the cranial arteries in the human embryo. Contrib Embryol 32: 205-262, 1948

10) Takano S, Saitoh M, Miyasaka Y, Yada K, Takagi H: Fenestration of the intracranial internal carotid artery. Neurol Med Chir (Tokyo) 31: 740-742, 1991

11) Tripathi M, Goel V, Padma MV, Jain S, Maheshwari MC, Gaikwad S, Gupta V, Chandra PS, Mehta VS: Fenestration of the posterior communicating artery. Neurol India 51: 75-76, 2003

12) Yasargil MG: Microneurosurgery I. Stuttgart, Georg Thieme Verlag, 1984, pp 60-66

13) Yock DH: Fenestration of the supraclinoid internal carotid artery with rupture of associated aneurysm. AJNR Am J Neuroradiol 5: 634-636, 1984

Address reprint requests to: Shiro Baba, M.D., Department of Neurosurgery, Nagasaki University Graduate School of Biomedical Sciences, 1-7-1 Sakamoto, Nagasaki 852-8501, Japan. e-mail: shishi14@nagasaki-u.ac.jp 